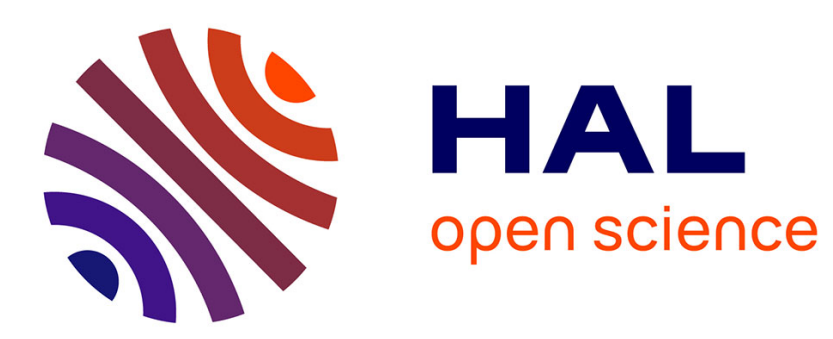

\title{
A symmetry for the widths of the eigen value spectra of Boson and Fermion systems

\author{
V.K.B. Kota
}

\section{To cite this version:}

V.K.B. Kota. A symmetry for the widths of the eigen value spectra of Boson and Fermion systems. Journal de Physique Lettres, 1979, 40 (22), pp.579-582. 10.1051/jphyslet:019790040022057900 . jpa00231692

\section{HAL Id: jpa-00231692 https://hal.science/jpa-00231692}

Submitted on 1 Jan 1979

HAL is a multi-disciplinary open access archive for the deposit and dissemination of scientific research documents, whether they are published or not. The documents may come from teaching and research institutions in France or abroad, or from public or private research centers.
L'archive ouverte pluridisciplinaire HAL, est destinée au dépôt et à la diffusion de documents scientifiques de niveau recherche, publiés ou non, émanant des établissements d'enseignement et de recherche français ou étrangers, des laboratoires publics ou privés. 


\title{
A symmetry for the widths of the eigen value spectra of Boson and Fermion systems
}

\author{
V. K. B. Kota \\ Physical Research Laboratory Navrangpura, Ahmedabad-380 009, India \\ (Reçu le 12 juillet 1979, accepté le ler octobre 1979)
}

\begin{abstract}
Résuméj-L'identité de Nomura $N \rightarrow-N$ pour la largeur des spectres de valeurs propres de systèmes de bosons et de fermions est étendue au sous-espace des quasi-spins. Nous montrons qu'elle est valable même pour les largeurs partielles dans ce sous-espace. Ce résultat ouvre la voie à l'extension aux systèmes de bosons des résultats connus pour les systèmes de fermions. D'autre part, il permet d'obtenir une classe de coefficients de Wigner de $\mathrm{SU}(N) \supset 0(N)$ à partir de ceux de $\mathrm{SU}(N) \supset \mathrm{Sp}(N)$.
\end{abstract}

\begin{abstract}
The Nomura's identity [Ann. Phys. 91 (1975) 83] $N \rightarrow-N$ for the widths of the eigen value spectra in scalar space of Boson and Fermion systems is shown to be valid for their quasispin subspaces also. We further show that it is valid even for their partial widths in quasispin subspaces. This result, besides paving a way to obtain the results for Boson systems from the known results for Fermion systems, gives us a way to obtain a class of $\mathrm{SU}(N) \supset 0(N)$ Wigner coefficients from $\mathrm{SU}(N) \supset \mathrm{Sp}(N)$ Wigner coefficients.
\end{abstract}

In recent years a lot of effort has gone in Nuclear Physics, into the development of the propagation methods [1] for widths and partial widths of eigenvalue spectra in various spectroscopic spaces. A knowledge of widths allows us to study various properties of many particle systems $[1,2]$ if the eigenvalue spectra are normal, as in the case of nuclear spectra. While a knowledge of the partial widths allows us to study the correctness of various group symmetries $[3,4]$ or truncation procedures. All the work reported in nuclear physics literature is restricted to the development of propagation methods for Fermion systems. The development of the same for Boson systems was initiated by Nomura [5]. He obtained expressions for the widths in scalar space of Boson systems. In the process he noticed that his expressions will go into their Fermion counterparts by simply changing $N \rightarrow-N$ ( $N$ is the number of single particle states) and taking modulo of the final result. Recently we found [6] that this simple symmetry relationship holds good even for the third and fourth moments, for interacting as well as for non-interacting Bosons. Although in ref. [7] analytic formulas for the first four moments of the eigenvalue spectra for noninteracting Bosons are given, the authors could not find this simply symmetry relation $N \rightarrow-N$ as they fail to simplify their formulas. Similarly in ref. [8]
Nomura might have noticed it for interacting Bosons if he dealt with irreducible (IR) $K$-body operators instead of $K$-body operators having particle hole symmetry. So far all the work on Boson systems is limited to scalar space. However one can learn more about the structure of the eigenfunction by decomposing the scalar space into various subspaces and study the partial widths. Here we are making a beginning by studying the widths and partial widths for Boson system with quasispin (seniority) decomposition of the scalar space. We demonstrate that the $N \rightarrow-N$ symmetry holds good for quasispin subspaces also (even for the partial widths).

We consider a system of $m$ Fermions occupying $N$-single particle states. Then the scalar space corresponds to the space spanned by $\left\{1^{m}\right\}$ partition of $\mathrm{U}(N)$ group. Similarly for $m$ Bosons it is $\{m\}$ partition of $\mathrm{U}(N)$ group. We restrict ourselves to $1+2$ body Hamiltonians $(H)$ throughout this article. The width produced by the IR 1-body part of $H$ (the IR $k$-body part transform according to $\left\{2^{k} 1^{N-k}\right\}$ partition of $\mathrm{U}(N)$ for Fermions and $\left\{2 k, K^{N-2}\right\}$ partition for Bosons) in the scalar space is given as

$$
\sigma^{2}(m)=\frac{m(N-m)}{N(N-1)}\langle\langle\operatorname{IR} 1 \text {-body }\rangle\rangle^{1}
$$


for Fermions and for Bosons

$$
\sigma^{2}(m)=\frac{m(N+. m)}{N(N+1)}\left\langle\langle\text { IR 1-body }\rangle^{1}\right.
$$

where \langle\rangle$^{1}$ is the trace in 1-particle scalar space. It is trivial to see that eq. (2) can be obtained from eq. (1) by changing $N \rightarrow-N$ and taking the modulo of the final result.

Similarly for IR 2-body operators we have,

$$
\begin{array}{r}
\sigma^{2}(m)=\frac{m(m-1)(N-m)(N-m-1)}{N(N-1)(N-2)(N-3)} \\
\ll \text { IR 2-body }\rangle^{2}
\end{array}
$$

for Fermions and, for Bosons

$$
\begin{array}{r}
\sigma^{2}(m)=\frac{m(m-1)(N+m)(N+m+1)}{N(N+1)(N+2)(N+3)} \\
\ll \text { IR 2-body }\rangle^{2} .
\end{array}
$$

Now we will proceed to the quasispin decomposition of the scalar space. This corresponds to the subgroup decomposition $\mathrm{U}(N) \supset \mathrm{Sp}(N)$ for Fermions and $\mathrm{U}(N) \supset 0(N)$ for Bosons. The complementary group [9] for the $\mathrm{Sp}(N)$ group in the above chain is $\mathrm{SU}(2)$ whose quantum number $v(v$ is the seniority quantum number) can be used to label the IR representations $\langle v\rangle$ of $\operatorname{Sp}(N)$. For $m$ Fermions, $v=m$, $m-2, \ldots, 0$ or 1 . Similarly for the $0(N)$ group, the complementary group is $\mathrm{SU}(1,1)$ and its quantum numbers $v, v=m, m-1, \ldots, 0$ or 1 can be used to label the IR representations $[v]$ of $0(N)$. The width $\sigma^{2}(m, v)$ of the IR representation [ $\left.v\right]$ is defined as

$$
\sigma^{2}(m, v)=\sum_{\alpha}\left\langle m v \alpha\left|\tilde{H}^{2}\right| m v \alpha\right\rangle / \mathrm{d}(v)
$$

where $\mathrm{d}(v)$ is the dimensionality of the representation [v] and $\tilde{H}$ is the traceless part of $H$. The width $\sigma^{2}(m, v)$ can be split into partial widths, the internal width $\sigma^{2}(m, v \rightarrow v)$ and the external widths $\sigma^{2}\left(m, v \rightarrow v^{\prime}\right)$, $v \neq v^{\prime}$ as

where

$$
\sigma^{2}(m, v)=\sum_{v^{\prime}} \sigma^{2}\left(m, v \rightarrow v^{\prime}\right)
$$

$\sigma^{2}\left(m, v \rightarrow v^{\prime}\right)=\sum_{\alpha, \beta}\left|\left\langle m v \alpha|\tilde{H}| m v^{\prime} \beta\right\rangle\right|^{2} / \mathrm{d}(v)$.

Quesne and Spitz $[4,10]$ developed methods to obtain widths and partial widths for quasispin subspaces of Fermion systems. We follow the same method for Bosons. However here one has to use the properties of the SU(1,1) group (see ref. [11] for details of this Boson quasispin group). We decomposed $H$ according to IR tensors of $\operatorname{SU}(1,1)$ group as done by Arima and Ichimura for Fermions [12]. We also decomposed $H$ into IR tensors of $\mathrm{U}(N) \supset 0(N)$ chain. Then we obtain the following IR tensors

$$
\begin{aligned}
\mathbf{H}=\mathbf{H}^{\{0\}[0]}+\mathbf{H}^{\left\{21^{N-2}\right\}[2]}+\mathbf{H}^{\left\{42^{N-2}\right\}[2]} \underset{\alpha=1}{[2]} & +\mathbf{H}^{\left\{42^{N-2}\right\}[2]}+\mathbf{H}^{\left\{2^{N-2}\right\}[4]} \\
& +\mathbf{H}^{\left\{42^{N-2}\right\}\left[2^{2}\right]} .
\end{aligned}
$$

The label $\alpha$ is used to distinguish the two IR representations [2] occuring in the reduction of $\left\{42^{N-2}\right\}$ partition. In the above decomposition the first tensor gives the centroid, the last tensor gives only the internal width, while the rest will produce both internal as well as external widths. For a single shell only the first and the last two tensors survive, while for a pure one body interaction only the first two tensors survive.

We can calculate the widths in quasispin space by writing a trace equivalent for $H^{2}$ in terms of $m$, the particle number and the pairing operator $P_{2}$. Knowing the widths $\sigma^{2}\left(t, v_{0}\right)$ in $0-4$ particle space, we obtain

$$
\sigma^{2}(m, v)=\sum_{\substack{t=0 \\
v_{0} \in t}}^{4}\left(\begin{array}{c}
4-m \\
4-t
\end{array}\right) \operatorname{PR}\left(t, v_{0}\right) \sigma^{2}\left(t, v_{0}\right)
$$

where $\operatorname{PR}(0,0)=1, \quad \operatorname{PR}(1,1)=m, \quad \operatorname{PR}(2,0)=P_{2} / \Omega, \quad \operatorname{PR}(2,2)=\left(\begin{array}{c}m \\ 2\end{array}\right)-P_{2} / \Omega, \quad \operatorname{PR}(3,1)=\frac{m-1}{\Omega+1} P_{2}$,

$$
\begin{gathered}
\operatorname{PR}(3,3)=\left(\begin{array}{c}
m \\
3
\end{array}\right)-\frac{m-2}{\Omega+1} P_{2}, \quad \operatorname{PR}(4,0)=P_{2}\left(P_{2}-m+\Omega+2\right) / 2 \Omega(\Omega+1), \\
\operatorname{PR}(4,2)=P_{2}\left\{\left(\begin{array}{c}
m-2 \\
2
\end{array}\right)-\frac{1}{\Omega}\left(P_{2}-m+\Omega+2\right)\right\} \mid \Omega+2
\end{gathered}
$$

and

$$
\operatorname{PR}(4,4)=\left(\begin{array}{c}
m \\
4
\end{array}\right)-\frac{(m-2)(m-3)}{2(\Omega+1)} P_{2}+\frac{P_{2}\left(P_{2}+\Omega+2-m\right)}{2(\Omega+1)(\Omega+2)} .
$$

Here $\Omega=N / 2$ and $\left\langle m v \alpha\left|P_{2}\right| m v \alpha\right\rangle=\left\langle P_{2}\right\rangle^{m, v}=(m-v)(2 \Omega+m+v-2) / 4$. By $\Omega \rightarrow-\Omega$ transformation, $\left\langle P_{2}\right\rangle_{\text {Bosons }}^{m, v} \rightarrow-\left\langle P_{2}\right\rangle_{\text {Fermions }}^{m, v}$ and it leads to the result that all $\operatorname{PR}\left(t, v_{0}\right)$ given above for Bosons 
will go into their Fermion counterparts (see eq. (A.1) of ref. [13]) by $\Omega \rightarrow-\Omega$ transformation, here we need not take modulo. Hence the total width in Boson quasispin space can be obtained from that of the corresponding Fermion quasispin space width by just changing $\Omega \rightarrow-\Omega$. The quasispin tensor decomposition together with the $\mathrm{U}(N) \supset 0(N)$ tensor decomposition of $\mathbf{H}$ allows us to obtain explicit analytic expressions for partial widths when we make use of the methods of ref. [10]. Now we clearly see that the $N \rightarrow-N$ symmetry persists even for the partial widths in quasispin space. We can understand this interesting result using some simple arguments as given below.

Let us consider a pure one body Hamiltonian. Then only the IR tensor [2] will produce partial widths by mixing $[v]$ and $[v \pm 2]$ partitions. We obtain the partial widths $\sigma_{[2]}^{2}(m, v \rightarrow v-2)$ by writing a recursion relation

$$
\begin{aligned}
\sigma^{2}(v, v \rightarrow v-2)=[\mathrm{d}(v-2) / \mathrm{d}(v)] *\left\{\sigma^{2}(v, v-2)-f^{2}(v, v-2) \sigma^{2}(v-2, v-2)+\right. \\
\\
{\left.\left[f^{2}(v, v-2)-g^{2}(v, v-2)\right] \sigma^{2}(v-2, v-2 \rightarrow v-4)\right\} }
\end{aligned}
$$

and

$$
\sigma^{2}(m, v \rightarrow v-2)=g^{2}(m, v) \sigma^{2}(v, v \rightarrow v-2) .
$$

The dimension factor $\mathrm{d}(v)$ has values,

$\mathrm{d}(v)=\frac{\Omega+v-1}{\Omega-1}\left(\begin{array}{c}2 \Omega+v-3 \\ v\end{array}\right)$ for Bosons and $\mathrm{d}(v)=\frac{\Omega-v+1}{\Omega+1}\left(\begin{array}{c}2 \Omega+2 \\ v\end{array}\right)$ for Fermions.

They have clearly $\Omega \rightarrow-\Omega$ symmetry (together with modulo). Moreover, $\mathrm{d}(v-2) / \mathrm{d}(v)$ for Bosons will go into its Fermion counterpart without taking the modulo. The $f$ and $g$ factors are ratios of $\operatorname{SU}(1,1)$ Wigner coefficients, for Bosons.

$$
\begin{aligned}
g^{2}(m, v) & =\left[\begin{array}{llll}
\langle(\Omega+v-2) / 2 & (m+\Omega) / 2 & 10 \|(\Omega+v) / 2 & (m+\Omega) / 2\rangle \\
\hline\langle(\Omega+v-2) / 2 & (v+\Omega) / 2 & 10 \|(\Omega+v) / 2 & (v+\Omega) / 2\rangle
\end{array}\right]^{2} \\
& =(m-v+2)(2 \Omega+m+v-2) / 4(\Omega+v-1)
\end{aligned}
$$

and it goes into its Fermion counterpart (see eq. (4.33) of ref. [4]) by $\Omega \rightarrow-\Omega$ transformation without taking the modulo. Similarly

$$
f(m, v)=(\Omega+m / \Omega+v)^{2}
$$

goes into its Fermion counterpart (see eq. (4.34) of ref. [4]) by $\Omega \rightarrow-\Omega$ transformation without taking the modulo. We have demonstrated earlier that $\sigma^{2}$ has $\Omega \rightarrow-\Omega$ symmetry. Hence in the above recursion formula all factors are the same for Fermions and Bosons except that $\Omega \rightarrow-\Omega$ and $\Omega$ is a pure number. Hence the solution of the recursion expression will be the same for Bosons and Fermions except that $\Omega \rightarrow-\Omega$. So the partial width $\sigma_{[2]}^{2}(m, v \rightarrow v-2)$ for single particle energies will have $\Omega \rightarrow-\Omega$ symmetry. Now using eq. (4.17) of ref. [10] we can obtain the $\mathrm{U}(N)$ Wigner coefficient

$$
\left|\left\langle\{m\}[v]\left\{21^{N-2}\right\}[2] \|\{m\}[v-2]\right\rangle\right|^{2} .
$$

For a $1+2$ body $H$,

$$
H=\sum_{i} \varepsilon_{i} \eta_{i}+\frac{1}{2} \sum_{i \leqslant j, \mathbf{K} \leqslant l} W_{i j \mathrm{~K} l}^{L} a_{i}^{+} a_{j}^{+} a_{\mathrm{K}} a_{l},
$$

the width produced by the tensor part which transforms according to the IR representation [2] of $0(N)$ will have contributions from single particle energies as well as two body matrix elements. Here we cannot use the recursion relations of ref. [4] as they use particle-hole symmetry. However a knowledge of $\mathrm{SU}(1,1)$ Wigner coefficients allows us to obtain directly $\sigma_{[2]}^{2}(m, 2 \rightarrow 0)$, without using them. The result is

$$
\sigma_{[2]}^{2}(m, 2 \rightarrow 0)=\frac{m(2 \Omega+m)}{(2 \Omega-1)(\Omega+1)^{2}} \frac{1}{\Omega} \sum_{i} \Omega_{i} \lambda_{i}^{2}(m)
$$

where

$$
\begin{aligned}
\lambda_{i}(m) & =\bar{\varepsilon}_{i}+\frac{m-2}{\Omega+2} \overline{\hat{\varepsilon}}_{i}+\frac{\Omega+m}{\Omega+2} \bar{\varepsilon}_{i} \\
\dot{\varepsilon}_{i} & =\frac{1}{2} \sum_{j} W_{i j i j}^{L=0} \sqrt{\frac{\Omega_{j}}{\Omega_{i}}}
\end{aligned}
$$

and

$$
\hat{\varepsilon_{i}}=\frac{1}{4 \Omega_{i}} \sum_{j} W_{i j i j}^{L}(2 L+1)\left(1+\delta_{i j}\right) .
$$

$\bar{\varepsilon}_{i}$ are traceless single particle energies,

$$
\bar{\varepsilon}_{i}=\varepsilon_{i}-\frac{1}{\Omega} \sum_{i} \Omega_{i} \varepsilon_{i}
$$

and $\Omega_{i}$ is the degeneracy of the $i$ th orbit. The above expression together with the $\mathrm{U}(N) \supset 0(N)$ Wigner coefficients evaluated by considering pure single particle energies, now yield 


$$
\sigma_{[2]}^{2}(m, v \rightarrow v-2)=\frac{\Omega(v-1) v(m-v+2)(2 \Omega+m+v-2)}{2(\Omega+1)(2 \Omega-1)(\Omega+v-1)(\Omega+v-2)} \frac{1}{\Omega} \sum_{i} \Omega_{i} \lambda_{i}^{2}(m) .
$$

It is straight-forward to see that this partial width has $\Omega \rightarrow-\Omega$ symmetry. In ref. [13] the corresponding expressions for Fermions are given. Note that the induced single particle energies $\frac{m-2}{\Omega+2} \hat{\varepsilon}_{i}$ and $\frac{\Omega+m}{\Omega+2} \dot{\varepsilon}_{i}$ also have $\Omega \rightarrow-\Omega$ symmetry (here we have to take the modulo).

Finally to obtain the partial widths produced by the $T^{\left\{\mathbf{4 2}^{N-2}\right\}[4]}$ tensor part, we consider a two-body interaction in a single shell. As a two-body interaction here behaves as a quasispin tensor of rank 2 , we can write a recursion relation for the partial widths, $\sigma_{[4]}^{2}(m, v \rightarrow v-2)$ and $\sigma_{[4]}^{2}(m, v \rightarrow v-4)$. Now applying the same reasoning that we adopted for obtaining the partial widths produced by pure single particle energies, we obtain the $\mathrm{U}(N) \supset 0(N)$ Wigner coefficients

$$
\left|\left\langle\{m\}[v]\left\{42^{N-2}\right\}[4] \|\{m\}[v-2]\right\rangle\right|^{2} \text { and }\left|\left\langle\{m\}[v]\left\{42^{N-2}\right\}[4] \|\{m\}[v-4]\right\rangle\right|^{2} \text {. }
$$

These coefficients will go into their Fermion counterparts by $\Omega \rightarrow-\Omega$ transformation. Using them we obtain, for a 2-body Hamiltonian operating in many orbits,

$$
\begin{aligned}
& \dot{\sigma}_{[4]}^{2}(m, v \rightarrow v-2)= \\
& \quad=\sigma_{[4]}^{2}(2) * \frac{3(\Omega-1) v(v-1)(v-2)(2 \Omega+v-2)(\Omega+m)^{2}(m-v+2)(2 \Omega+m+v-2)}{8(\Omega+2)(\Omega+3)(2 \Omega-1)(\Omega+v)(\Omega+v-1)(\Omega+v-2)(\Omega+v-4)}
\end{aligned}
$$

and

$$
\begin{aligned}
& \sigma_{[4]}^{2}(m, v \rightarrow v-4)= \\
& \quad=\sigma_{[4]}^{2}(2) * \frac{3 v(v-1)(v-2)(v-3)(m-v+2)(m-v+4)(2 \Omega+m+v-2)(2 \Omega+m+v-4)}{32(\Omega+3)(2 \Omega-1)(\Omega+v-1)(\Omega+v-2)(\Omega+v-3)(\Omega+v-4)} .
\end{aligned}
$$

The above expressions go into their Fermion counterparts by transformation.

Here we showed that all the external widths as well as the total width for the quasispin subspace of interacting as well as non-interacting Boson systems will go into their Fermion counter parts by $\Omega \rightarrow-\Omega$ transformation. Hence the internal widths will also have $\Omega \rightarrow-\Omega$ symmetry. The partial widths given in eqs. (12), (13) and (14) are of immediate use in studying the correctness of the $U(6) \supset 0(6)$ chain [14] of the Interacting Boson Approximation model (IBA) introduced recently to study transitional nuclei. The fact that $N \rightarrow-N$ symmetry holds good for scalar space leads to the same result for configuration space needs no explanation. Hence our present result leads to the fact that the $N \rightarrow-N$ symmetry extends even to the configuration-seniority subspaces. This is useful in studying the correctness of the IBA chain $\mathrm{U}(6) \supset \mathrm{U}(5) \supset 0(5)$ [15].
One very important outcome of the $N \rightarrow-N$ symmetry is that it allows us to obtain a class of $\mathrm{U}(N) \supset 0(N)$ Wigner coefficients from that of the $\mathrm{U}(N) \supset \mathrm{Sp}(N)$ decomposition. Here we have to use eq. (4.7) of ref. [10]. Hence we can translate all the coefficients tabulated in ref. [10] to their $\mathrm{U}(N) \supset 0(N)$ counterparts by changing $\Omega \rightarrow-\Omega$. These analytic formulas can be used even for $0(\Omega)$, where $\Omega$ is odd. The correspondence is as follows :

$$
\left\{1^{m}\right\} \rightarrow\{m\},\langle f\rangle \rightarrow[\tilde{f}],
$$

and

$$
\left\{2^{K} 1^{N-r}\right\} \rightarrow\left\{r,(r-k)^{N-2}\right\} .
$$

Making use of the work of Nomura [16] on higher moments in seniority space, we can evaluate many more $\mathrm{U}(N) \supset 0(N)$ coefficients.

\section{References}

[1] Chang, F. S., French, J. B. and Thio, T. H., Ann. Phys. N.Y. 66 (1971) 137.

[2] Draayer, J. P., French, J. B. and Wong, S. S. M., Ann. Phys. N.Y. 106 (1977) 472, 503.

[3] HeCht, K. T. and DraAyer, J. P., Nucl. Phys. A 223 (1974) 285.

[4] Quesne, C. and Spitz, S., Ann. Phys. N.Y. 85 (1974) 115.

[5] Nomura, M., Ann. Phys. N.Y. 91 (1975) 83.

[6] Kota, V. K. B. and Potbhare, V. (to be published).

[7] Bortignon, P. F., MANFredi, V. R. and SPITZ, S., Lett. Nuovo Cimento 23 (1978) 210.

[8] Nomura, M., Prog. Theor. Phys. 59 (1978) 1199.
[9] Moshinsky, M. and Quesne, C., J. Math. Phys. 11 (1970) 1631 .

[10] Quesne, C. and Sprtz, S., Ann. Phys. N.Y. 112 (1978) 304.

[11] UI, I., Ann. Phys. N.Y. 49 (1968) 69.

[12] Arima, A. and Ichimura, M., Prog. Theor. Phys. 36 (1966) 296.

[13] Quesne, C., Phys. Lett. 57B (1975) 422.

[14] Arima, A. and Iachello, F., Phys. Rev. Lett. 40 (1978) 385.

[15] Arima, A. and Iachello, F., Ann. Phys. N.Y. 99 (1976) 253.

[16] Nomura, M., Prog. Theor. Phys. 51 (1974) 489. 